\title{
Expression of mTOR and downstream signalling components in the JEG-3 and BeWo human placental choriocarcinoma cell lines
}

\author{
DIONISIS MPARMPAKAS ${ }^{1}$, ELENA ZACHARIADES ${ }^{1}$, HELEN FOSTER $^{1}$, ANJELI KARA ${ }^{1}$, \\ AMANDA HARVEY ${ }^{1}$, ANASTASIA GOUMENOU ${ }^{1,2^{*}}$ and EMMANOUIL KARTERIS ${ }^{1 *}$ \\ ${ }^{1}$ Centre for Cell Chromosome Biology, Biosciences, School of Health Sciences and Social Care, \\ Brunel University, Uxbridge UB8 3PH, UK; ${ }^{2}$ University of Crete, Division of Medicine, \\ Department of Obstetrics and Gynecology, Heraklion, Crete, Greece
}

Received February 27, 2009; Accepted April 7, 2009

DOI: 10.3892/ijmm_00000314

\begin{abstract}
Emerging data suggest that nutritional status and body weight are related to reproductive function, and nutrient imbalances during pregnancy lead to changes in the expression of fetal genes. Recent studies show that the mTOR acts as a placental growth signalling sensor and its expression is downregulated in intrauterine growth restriction. To date, very little is known about the expression of this signalling pathway in choriocarcinoma, one of the most lethal germ cell cancers. In this study, cultures of fusigenic (BeWo) and non-fusigenic (JEG-3) human choriocarcinoma cell lines were used to investigate the expression of mTOR and its downstream signalling components. The effects of an inducer of syncytialisation (forskolin) on mTOR, eIF4E binding proteins (4EBPs) and ribosomal protein $\mathrm{S} 6$ kinases (S6Ks) in BeWo cells were also assessed. RT-PCR studies revealed that mTOR, 4EBP and S6Ks are expressed at mRNA level in both JEG-3 and BeWo cells. Semi-quantitative RT-PCR analysis revealed that in early stages of syncytialisation $(50 \mu \mathrm{M}$ forskolin for $48 \mathrm{~h}$ ), the expression of mTOR and 4EBP was down-regulated when compared to unstimulated cells. In fully syncytialised cells $(50 \mu \mathrm{M}$ forskolin for $72 \mathrm{~h})$ the expression of both genes was similar to basal levels. Interestingly, the phosphorylation (Ser371, Thr389) status of p70S6K remained unaltered upon forskolin treatment. These data validate BeWo cells as an experimental model to study the effects of forskolin-induced syncytialisation on mTOR signalling.
\end{abstract}

Correspondence to: Dr Emmanouil Karteris, Centre for Cell Chromosome Biology, Biosciences, School of Health Sciences and Social Care, Brunel University, Uxbridge UB8 3PH, UK

E-mail: emmanouil.karteris@brunel.ac.uk

*Contributed equally

Key words: placenta, syncytialisation, mTOR, S6 Kinase, eIF4E binding proteins

\section{Introduction}

mTOR functions by integrating extracellular signals (growth factors and steroid hormones) with amino acid availability and intracellular energy status to control translation rates and additional metabolic processes (1). mTOR enhances translation initiation, in part by phosphorylating two major targets that cooperate to regulate translation initiation rates, the eIF4E binding proteins (4EBPs) and the ribosomal protein S6 kinases (S6K1 and 2) (2). It is now believed that mTOR also functions as a 'molecular switch' through S6 kinase depending on the status of the cell (3).

There are two isoforms of S6 kinase, $\mathrm{S} 6 \mathrm{~K} \alpha$ and $\beta$ (or S6K1 and 2), which have cytoplasmic (S6K $\alpha$ II and S6KßII) and nuclear ( $66 \mathrm{~K} \alpha \mathrm{I}$ and $\mathrm{S} 6 \mathrm{~K} \beta \mathrm{I})$ variants derived from alternative splicing at the $\mathrm{N}$-terminus. $\mathrm{S} 6 \mathrm{~K} \alpha$ and $\beta$ have a high level of sequence similarity, with the greatest homology in the kinase and kinase extension domains; however, they differ significantly at their $\mathrm{N}$ - and C-terminal regulatory regions, sharing only 28 and $25 \%$ homology respectively (4). In spite of their apparent cellular localisation, it is becoming clearer that the regulation of S6K is more complex. All isoforms are involved in nuclearcytoplasmic shuttling which is regulated by their phosphorylation status resulting in the presence of activated S6K in different cellular localisations (5-6).

To date, few studies implicated mTOR in placental signalling. For example, in vitro outgrowth experiments showed that mTOR deficient blastocysts were severely impaired in their ability to form trophoblasts (7). mTOR expression is downregulated in restricted fetal growth (8), and also functions as a placental growth signalling sensor (9). Experiments using immortalised human trophoblast cells revealed that cell proliferation was induced in response to glucose or following activation of Tie-2 receptor, involving an mTOR signalling pathway (9). This is of increasing importance since nutrient imbalances during pregnancy may lead to changes in both reproductive function (10) and the expression of fetal genes (11).

Currently, and despite the implications of mTOR signalling on cancer, there is no data about the involvement of this 
pathway on gestational trophoblastic diseases (GTD). These diseases have key characteristics, the abnormal growth of chorionic tissues and differential expression of oncogenes and growth factors (12). GTDs are a diverse group of diseases including complete hydatidiform and invasive moles and choriocarcinoma and placental site trophoblastic tumours (13). Interestingly, choriocarcinoma resembles the complete mole, based on the expression of various oncogenes and growth factors (12).

In this study we tested the hypothesis that an inducer of syncytialisation affects the expression of mTOR signalling components. The specific objectives of this study were: a) assessment of the expression of mTOR, 4EBPs and the ribosomal protein S6Ks in two human placental choriocarcinoma cell lines (BeWo and JEG-3), b) investigation of the effects of inducer of syncytialisation (forskolin) on these genes in BeWo cells.

\section{Materials and methods}

Placental tissue. Labouring placental tissues were obtained from women delivering at term without any complications ( $>37$ weeks of gestation, $n=3$ ). After delivery, the maternal and fetal surfaces of the placenta were dissected, and fetal membranes were peeled away from the placenta. Placental samples were washed in PBS and immediately stored in RNALater at $-80^{\circ} \mathrm{C}$. Ethical approval was granted from the local ethics authority.

Cell culture. BeWo and JEG-3 cell lines were purchased from the European Collection of Cell Cultures (ECACC, Salisbury, UK). The cells were maintained at standard culture conditions of $5 \% \mathrm{CO}_{2}$ in air at $37^{\circ} \mathrm{C}$. BeWo cells were cultured in Ham F12 (Sigma, UK) containing 10\% heat-inactivated fetal bovine serum (FBS), and $0.5 \%$ penicillin-streptomycin, whereas JEG-3 cells were maintained in MEME (Sigma) containing $10 \%$ heat-inactivated FBS, and $0.5 \%$ penicillinstreptomycin, $0.5 \%$ L-glutamine, $0.5 \%$ sodium pyruvate and $0.5 \%$ MEM non-essential amino acids.

Syncytialisation of BeWo cells. Forskolin (Sigma, UK) was used as an inducer of syncytialisation in BeWo cytotrophoblast cells. Syncytialisation of BeWo cells was assessed with 50 and $100 \mu \mathrm{M}$ forskolin over a total period of $72 \mathrm{~h}$, renewing forskolin treatment every $24 \mathrm{~h}$.

RNA isolation, $c D N A$ synthesis and PCR. Total ribonucleic acid was isolated using an RNA extraction kit (Sigma), according to manufacturer's instructions. RNA concentration was determined by spectrophotometric analysis (NanoDrop; Thermo Scientific, UK) and agarose gel electrophoresis. RNA (500 ng) was reverse-transcribed into cDNA using $5 \mathrm{IU} / \mu \mathrm{l}$ RNase $\mathrm{H}$ reverse transcriptase (Invitrogen, Paisley, UK). PCR amplification was performed using Taq polymerase (Invitrogen) and oligonucleotide primers as previously described (14). Twenty-eight cycles were performed consisting of an initial denaturing step at $94^{\circ} \mathrm{C}$ for $30 \mathrm{sec}$, annealing at $60^{\circ} \mathrm{C}$ for $1 \mathrm{~min}$ and elongation at $72^{\circ} \mathrm{C}$ for $1 \mathrm{~min}$.

Immunofluorescent analysis. BeWo (treated and untreated) and JEG-3 cells were fixed in $4 \%$ paraformaldehyde for $10 \mathrm{~min}$ prior to washes in PBS and incubation with $10 \%$ bovine serum albumin (BSA) for $1 \mathrm{~h}$. Cells were incubated for $1 \mathrm{~h}$ with an mTOR antibody (Santa Cruz Biotechnology, USA) at a 1:100 dilution in $1 \% \mathrm{BSA} / \mathrm{PBS}$. Cells were then washed with PBS prior to an incubation with anti-rabbit IgG-fluorescein isothiocyanate (FITC)-conjugated antibody (Santa Cruz Biotechnology) for $1 \mathrm{~h}$. Slides were washed with PBS and mounted in Vectashield $^{\circledR}$ Mounting Medium (Vector labs) containing the dye 4,6-diamido-2-phenylindole (DAPI) to counterstain nuclei. Images were captured using a Plan Apo Neofluor 63X NA 1.25 oil objective (Zeiss) on a Zeiss Axiovert $200 \mathrm{M}$ microscope and viewed using AxioVision software. Images were taken with a particular exposure time.

Protein extraction from BeWo cells. BeWo cells were cultured to $80 \%$ confluency, and in the presence or absence of forskolin (50-100 $\mu \mathrm{M}$ up to $72 \mathrm{~h}$ ). Cells were then lysed using $200 \mu \mathrm{l}$ $1 \mathrm{X}$ Laemmli buffer (Sigma) and denatured for $5 \mathrm{~min}$ at $100^{\circ} \mathrm{C}$ before cooled on ice.

Western immunoblotting. Samples were separated on an SDS$10 \%$ polyacrylamide gel and the proteins were transferred to a nitrocellulose membrane. The membrane was blocked in TBS containing $5 \%$ dried milk powder (w/v) and $0.1 \%$ Tween-20, for $1 \mathrm{~h}$ at room temperature. After three washes with TBS- $0.1 \%$ Tween, the nitrocellulose membranes were incubated with primary antibodies against proliferating cell nuclear antigen (PCNA), pan-Cadherin, phosphorylated (serine/threonine) p70S6K, and GAPDH (Cell Signalling, Sigma). The primary antiserum was used at a 1:1000 dilution overnight at $4^{\circ} \mathrm{C}$. The membranes were then washed thoroughly for $30 \mathrm{~min}$ with TBS- $0.1 \%$ Tween, before incubation with the secondary antirabbit HRP-conjugated immunoglobulin (1:2000) for $1 \mathrm{~h}$ at room temperature and further washing for 30 min with TBS- $0.1 \%$ Tween. Antibody complexes were visualised as previously described (15).

The nitrocellulose membranes were then stripped, prior to incubation with GAPDH antibody. Briefly, pre-warmed stripping solution (ß-mercaptoethanol $100 \mathrm{mM}, 10 \%$ SDS, Tris $\mathrm{HCl} 62.5 \mathrm{mM}$ ) was applied to each membrane and incubated at $50^{\circ} \mathrm{C}$ for $15 \mathrm{~min}$. Fresh stripping solution was then added to each membrane and incubated at $50^{\circ} \mathrm{C}$ for $15 \mathrm{~min}$. The membranes were briefly washed with $\mathrm{dH}_{2} \mathrm{O}$ and then thoroughly washed with TBS- $0.1 \%$ Tween to ensure complete removal of $\beta$-mercaptoethanol from membranes before blocking solution and primary antibody were applied.

\section{Results}

Expression of mTOR signalling components. RT-PCR analysis revealed that BeWo and JEG-3 cells express mTOR, 4EBP and the S6 kinase isoforms $\alpha 1, \alpha 2, \beta 1$ and $\beta 2$ at mRNA level. PCR analyses for the above genes produced PCR products of $537 \mathrm{bp}$ for mTOR, $299 \mathrm{bp}$ for 4EBP, and $585 \mathrm{bp}, 480 \mathrm{bp}, 468 \mathrm{bp}$, $336 \mathrm{bp}$ for the $\alpha 1, \alpha 2, \beta 1$ and $\beta 2 \mathrm{~S} 6 \mathrm{~K}$ isoforms (Fig. 1A). These PCR products are consistent with those described previously (14). Immunofluorescent analysis using a specific antibody for mTOR revealed cytoplasmic localisation of mTOR in both cell lines (Fig. 1B). The staining of mTOR appeared granular and dispersed in nature. 
A

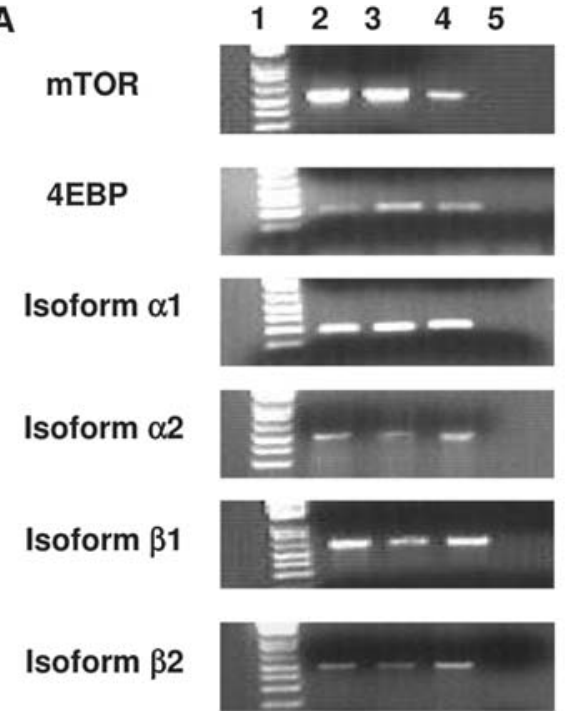

B
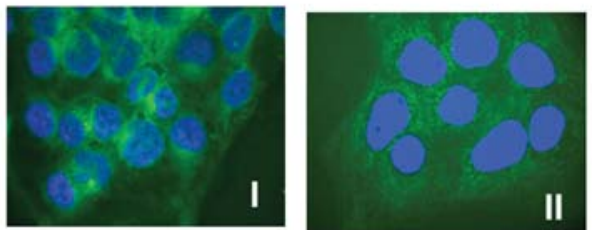

Figure 1. (A) Gene expression of mTOR, 4EBP and S6K isoforms. Lane 1, DNA ladder; lane 2, cDNA from human placenta; lane 3, cDNA from BeWo cells; lane 4, cDNA from JEG-3 cells; lane 5, negative control. (B) Immunofluorescent analysis of mTOR in BeWo (I) and JEG-3 (II) cells.

\section{A}

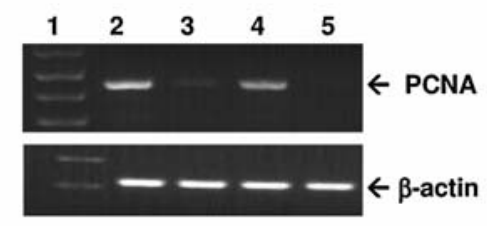

B

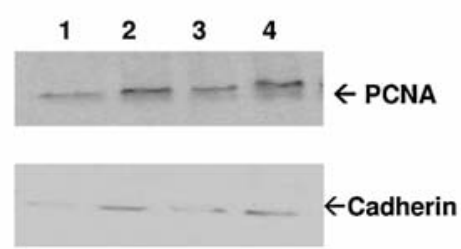

Figure 2. (A) Expression of PCNA mRNA in treated and untreated BeWo cells. Lane 1, DNA ladder; lane 2, cDNA from untreated BeWo cells (48 h); lane 3, cDNA from stimulated BeWo cells (50 $\mu \mathrm{M}$ forskolin, $48 \mathrm{~h})$; lane 4 , cDNA from untreated BeWo cells (48 h); lane 5, cDNA from stimulated BeWo cells $(100 \mu \mathrm{M}$ forskolin, $48 \mathrm{~h})$. (B) Protein expression of PCNA and Pan-Cadherin. Lane 1, BeWo cells treated with $50 \mu \mathrm{M}$ forskolin for $72 \mathrm{~h}$; lane 2, control, unstimulated BeWo cells ( $72 \mathrm{~h}$ ), lane 3 , BeWo cells treated with $100 \mu \mathrm{M}$ forskolin for $72 \mathrm{~h}$; lane 4 , control, unstimulated BeWo cells (72 h).

Syncytialisation of BeWo cells. In this experimental model, syncytialisation was achieved using forskolin. The syncytialisation model on BeWo cells was evaluated in detail, prior to elucidating the expression of mTOR signalling components in the differentiated state (syncytiotrophoblasts) of BeWo cells.

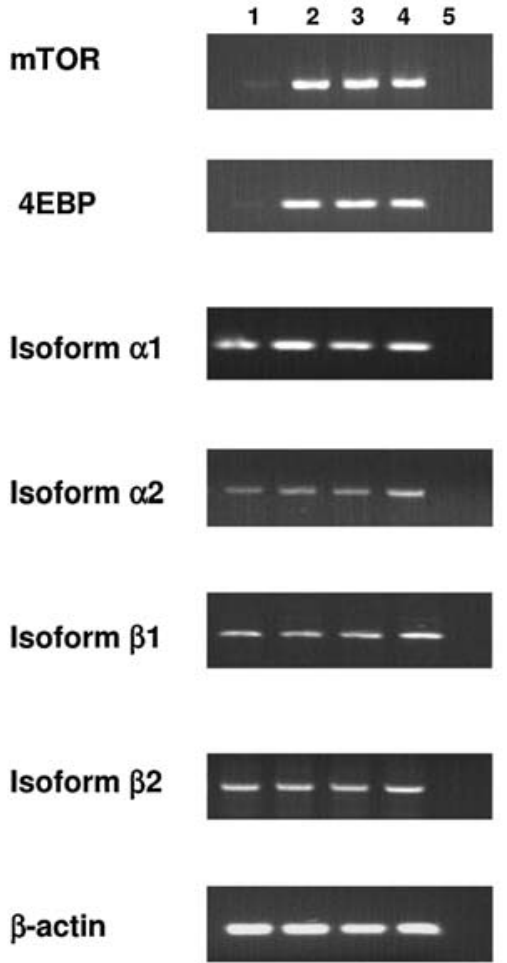

Figure 3. Effect of forskolin at $48 \mathrm{~h}$ on gene expression of mTOR signalling components. Lane 1, cDNA from stimulated BeWo cells $(50 \mu \mathrm{M}$ forskolin, $48 \mathrm{~h}$ ); lane 2, cDNA from untreated BeWo cells (control); lane 3, cDNA from stimulated BeWo cells (100 $\mu \mathrm{M}$ forskolin, $48 \mathrm{~h}$ ); lane 4, cDNA from untreated BeWo cells (control); lane 5, negative control.

BeWo cells treated with forskolin $(50$ and $100 \mu \mathrm{M})$ for $48 \mathrm{~h}$ revealed down-regulation of expression of PCNA at the mRNA level (Fig. 2A). At the protein level, the results of Western blotting demonstrated that after $72 \mathrm{~h}$ of exposure to either 50 or $100 \mu \mathrm{M}$ forskolin PCNA levels were down-regulated compared to controls (Fig. 2B). These data suggest that BeWo cells cease to proliferate, indicative of differentiation events taking place. Analysis of the cell membrane marker cadherin, was carried out to determine whether there was a decrease of the total plasma membrane in response to forskolin treatment. Decrease in plasma membrane levels is indicative of differentiation as cells become multinucleated during syncytialisation. Western blotting revealed down-regulation of cadherin protein levels in BeWo cells treated with forskolin for $72 \mathrm{~h}$ at both 50 and $100 \mu \mathrm{M}$ when compared with controls (Fig. 2B). Equal protein loading was ensured by stripping and blotting the nitrocellulose membranes with an antibody to the housekeeping gene GAPDH (data not shown).

Effect of syncytialisation on mTOR and downstream signalling components. Semi-quantitative RT-PCR analysis (comparing gene expression with the housekeeping gene $B$-actin) revealed that in early stages of syncytialisation $(50 \mu \mathrm{M}$ forskolin for $48 \mathrm{~h}$ ), the expression of mTOR and 4EBP was down-regulated when compared with basal unstimulated cells. However, in fully syncytialised cells ( $50 \mu \mathrm{M}$ forskolin for $72 \mathrm{~h}$ ) the gene expression of mTOR and 4EBP was unaltered (Figs. 3 and 4). mRNA expression of the isoforms $\mathrm{S} 6 \mathrm{~K} \alpha 1$ and 32 was unaffected by forskolin treatment at 48 and $72 \mathrm{~h}$, whereas $\alpha 2$ was down-regulated upon forskolin treatment $(100 \mu \mathrm{M})$ at 


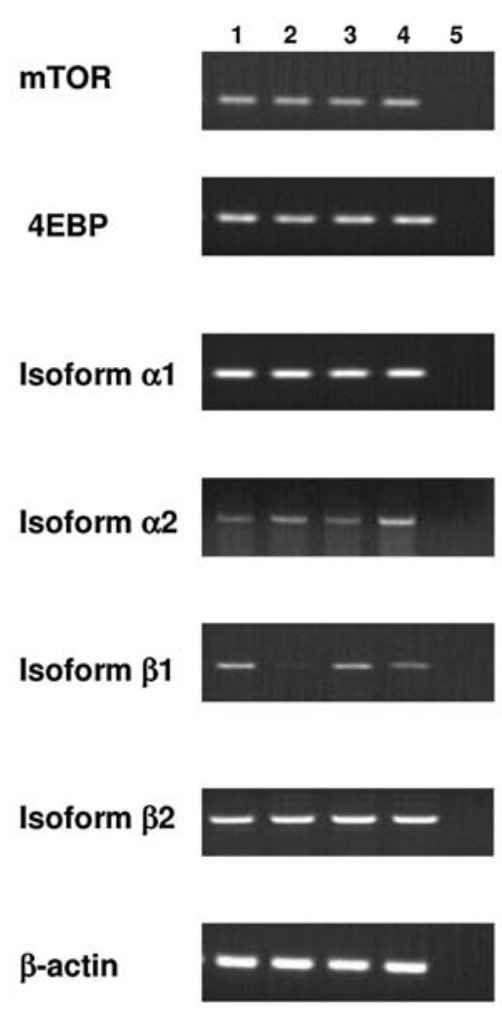

Figure 4. Effect of forskolin at $72 \mathrm{~h}$ on gene expression of mTOR signalling components. Lane 1, cDNA from stimulated BeWo cells (50 $\mu \mathrm{M}$ forskolin, $72 \mathrm{~h}$ ); lane 2, cDNA from untreated BeWo cells (control); lane 3, cDNA from stimulated BeWo cells (100 $\mu \mathrm{M}$ forskolin, $72 \mathrm{~h}$ ); lane 4, cDNA from untreated BeWo cells (control); lane 5, negative control.

$48 \mathrm{~h}$ (Figs. 3 and 4). Gene expression of $\alpha 2$ and $\beta 1$ was also altered at $72 \mathrm{~h}$, with $\alpha 2$ being down-regulated in fully syncytialised cells (100 $\mu \mathrm{M}$ forskolin) treatment, whereas $B 1$ was up-regulated with forskolin treatment (Fig. 4).

The protein expression of mTOR was assessed in syncytialised BeWo cells, using immunofluorescent analysis. Similar intense cytoplasmic staining was detected in unstimulated BeWo cells as previously described (Fig. 1B). Although this approach is not quantitative, the expression of mTOR at 48 and $72 \mathrm{~h}$ in both syncytialised and unstimulated BeWo cells was very similar in terms of the intensity of the immunostaining and the cellular localisation (Fig. 5).

The activity of p70S6K was assessed in stimulated and unstimulated BeWo cells by measuring the effect of forskolin on the phosphorylation status of S6K. Under basal conditions (i.e. no forskolin treatment), there was no change in the phosphorylation status of the p70S6K (Ser371, Thr 389) when we compared treated and untreated BeWo cells, and corrected over the housekeeping gene GAPDH at 48 or 72 h (Fig. 6).

\section{Discussion}

The two cell lines studied are widely used to investigate placental physiology and function. These cell lines have distinct fusigenic capacities. The JEG-3 cell line, despite being of a choriocarcinoma origin, is unable to morphologically differentiate and therefore it resembles the undifferentiated and hormonally inactive cytotrophoblast cells, making it an appropriate in vitro model to investigate first trimester placental events (16).

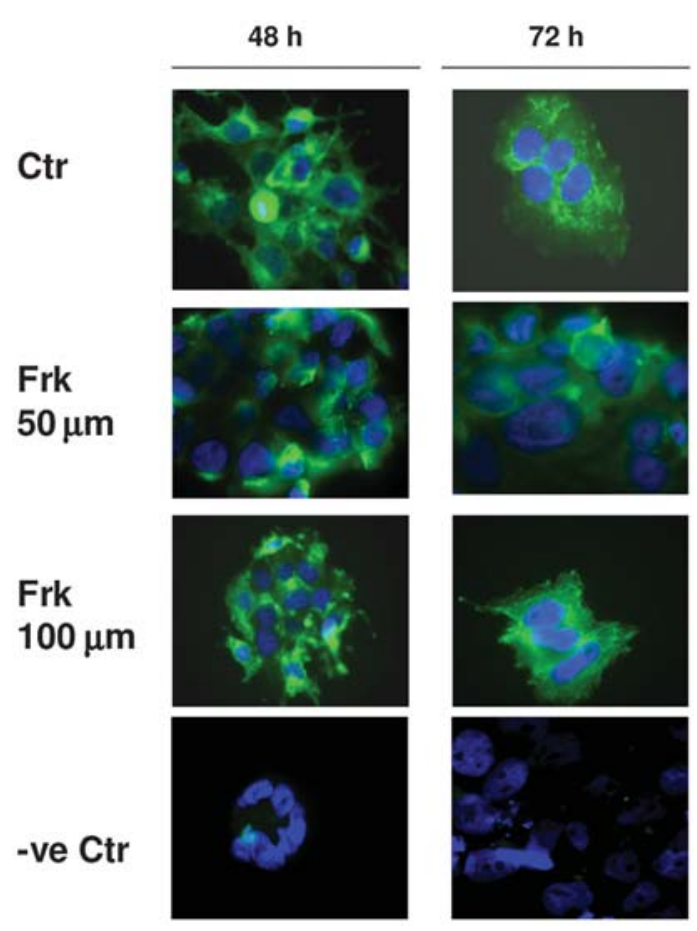

Figure 5. Cellular distribution of mTOR in BeWo cells treated with forskolin (Frk) at 48 and $72 \mathrm{~h}$.

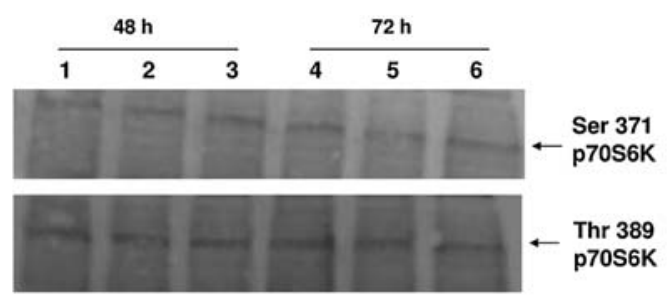

Figure 6. Effect of forskolin $(50$ and $100 \mu \mathrm{M})$ at 48 and $72 \mathrm{~h}$ on Ser371 and Thr389 phosphorylation of p70S6K. Lanes 1 and 4, control (unstimulated BeWo cells); lanes 2 and 5, BeWo cells treated with $50 \mu \mathrm{M}$ forskolin; lanes 3 and 6 , BeWo cells treated with $100 \mu \mathrm{M}$ forskolin.

BeWo cells are fusigenic and when they are fully differentiated (i.e. syncytialised) they resemble a third trimester placental model. At this stage the predominant feature of the human placenta is the hormonally active syncytiotrophoblast layer. The multinucleated syncytiotrophoblasts cover the external surface of the placental villous and are in direct interface with maternal blood (17). Undifferentiated BeWo cells are similar in morphology to primary trophoblast cultures, with a monolayer and microvillar projections on the apical side. The capability of BeWo cells to differentiate has established these cells as an in vitro model to study immune, endocrine and developmental aspects (18-20), as well as placental transport mechanisms (21). In this report we demonstrate for first time the expression of mTOR, p70S6K isoforms and 4EBP in these two cell lines and provide evidence of how the process of syncytialisation affects the expression of these components as well as the activity of p70S6K.

Immunofluorescent analysis revealed strong cytoplasmic staining for mTOR in both cell lines. This is in agreement with previous studies showing that mTOR is located chiefly in the cytoplasm $(8,14)$. 
Previous studies show that mTOR also shuttles between the nucleus and cytoplasm (22). The authors proposed that the nucleo-cytoplasmic shuttling of mTOR is required for the maximal activation of S6K1 (22). In our study, nuclear expression of mTOR under basal conditions in JEG-3 and undifferentiated BeWo was not observed. Even after forskolininduced syncytialisation, no apparent translocation of mTOR was noted in BeWo cells at either 48 or $72 \mathrm{~h}$. In relation to human placenta, mTOR protein is highly expressed within the cytosol of the syncytiotrophoblast (8). Future studies should determine the cellular localisation of mTOR in human placentae throughout pregnancy as well as in placentae from metabolically complicated pregnancies.

In our experimental model, treatment with forskolin induced a transient $(48 \mathrm{~h})$ decrease in the gene and protein expression of mTOR in BeWo cells, as well as a decrease in the gene expression of $4 \mathrm{EBP}$. These results support a recent study showing that forskolin altered the expression of several genes during differentiation of BeWo cells. For example, mRNA expression of SLPI, ELF-1 $\alpha-1$, prolyl 4-hydroxylase- $\beta$ and gastric associated differentially expressed protein was up-regulated after forskolin induced differentiation. In contrast, LMO-4 and Rab-5 interacting protein transcripts were down-regulated during cytotrophoblast differentiation (18).

Interestingly, despite changes in the gene expression of the p70S6K isoforms $\alpha 2$ and $\beta 1$ at $72 \mathrm{~h}$, the activity of this kinase in terms of phosphorylation remains unaltered throughout. It appears therefore that expression and phosphorylation of this enzyme is differentially regulated in BeWo cells. Indeed, the serine/threonine kinase p70S6K1 is also activated through mTOR-insensitive signalling pathways.

Microarray analysis demonstrated that a number of transcripts were differentially expressed between BeWo and JEG-3 cells (23). These distinct characteristics suggest that each cell line varies with regards to their ability to respond to certain stimuli, such as hormones and nutrients. As a result, downstream signalling cascades, including mTOR, might also be differentially regulated.

Collectively these novel data provide evidence that two human choriocarcinoma cell lines express mTOR and its downstream signalling components, and syncytialisation events affect gene expression of these components. In terms of using these experimental models to study placental mTOR signalling, it should be noted that there are differences in the expression of genes between an immortalised choriocarcinoma cell line and primary trophoblast cell cultures. Future studies, should map in detail these components during the first, second and third trimesters of pregnancy and expand observations in other critical intrauterine tissues such as fetal membranes, as well in other pathophysiological complications of pregnancy.

\section{Acknowledgements}

This study was supported by the Greek State Scholarships Foundation (IKY).

\section{References}

1. Hay N and Sonenberg N: Upstream and downstream of mTOR. Genes Dev 18: 1926-1945, 2004.

2. Petroulakis E, Mamane Y, Le Bacquer O, Shahbazian D and Sonenberg N: mTOR signaling: implications for cancer and anticancer therapy. Br J Cancer 94: 195-199, 2006.

3. Asnaghi L, Bruno P, Priulla M and Nicolin A: mTOR: a protein kinase switching between life and death. Pharmacol Res 50: 545-549, 2004.

4. Dufner A and Thomas G: Ribosomal S6 kinase signalling and the control of transaltion. Exp Cell Res 253: 100-109, 1999.

5. Panasyuk G, Nemazanyy I, Zhyvoloup A, et al: Nuclear export of S6K1 II is regulated by protein kinase CK2 phosphorylation at Ser-17. J Biol Chem 281: 31188-31201, 2006.

6. Valovka T, Verdier F, Cramer R, et al: Protein kinase C phosphorylates ribosomal protein S6 kinase betaII and regulates its subcellular localization. Mol Cell Biol 23: 852-863, 2003.

7. Gangloff YG, Mueller M, Dann SG, et al: Disruption of the mouse mTOR gene leads to early postimplantation lethality and prohibits embryonic stem cell development. Mol Cell Biol 24: 9508-9516, 2004

8. Roos S, Jansson N, Palmberg I, Säljö K, Powell TL and Jansson T: Mammalian target of rapamycin in the human placenta regulates leucine transport and is down-regulated in restricted fetal growth. J Physiol 582: 449-459, 2007.

9. Wen HY, Abbasi S, Kellems RE and Xia Y: mTOR: A placental growth signaling sensor. Placenta 26: S63-S69, 2005.

10. Lim SS, Noakes M and Norman RJ: Dietary effects on fertility treatment and pregnancy outcomes. Curr Opin Endo Diabetes Obes 14: 465-469, 2007

11. Bursztyn M and Ariel I: Maternal-Fetal deprivation and the cardiometabolic syndrome. J Cardiometab Syndr 1: 141-145, 2006.

12. Fulop V, Mok SC and Berkowitz RS: Molecular biology of gestational trophoblastic neoplasia: a review. J Reprod Med 49: 415-422, 2004

13. Cheung AN: Pathology of gestational trophoblastc diseases. Best Pract Res Clin Obstet Gynaecol 7: 849-868, 2003.

14. Liu Y, Hidayat S, Su WH, Deng X, Yu DH and Yu BZ: Expression and activity of mTOR and its substrates in different cell cycle phases and in oral squamous cell carcinomas of different malignant grade. Cell Biochem Funct 25: 45-53, 2007.

15. Karteris E, Grammatopoulos D, Randeva H and Hillhouse EW: Signal transduction characteristics of the corticotropin-releasing hormone receptors in the feto-placental unit. J Clin Endocrinol Metab 85: 1989-1996, 2000

16. Ntrivalas E, Kwak-Kim J, Beaman K, Mantouvalos H and Gilman-Sachs A: An in vitro coculture model to study cytokine profiles of natural killer cells during maternal immune celltrophoblast interactions. J Soc Gynecol Investig 13: 196-202, 2006.

17. Das M, Xu B, Lin L, Chakrabarti S, Shivaswamy V and Rote NS Phosphatidylserine efflux and intercellular fusion in a BeWo model of human villous cytotrophoblast. Placenta 25: 396-407, 2004.

18. Neelima PS and Rao AJ: Gene expression profiling during Forskolin induced differentiation of BeWo cells by diferential display RT-PCR. Mol Cell Endocrinol 281: 37-46, 2008.

19. Ellinger I, Schwab M, Stefanescu A, Hunziker W and Fuchs R: IgG transport across trophoblast-derived BeWo cells: a model system to study IgG transport in the placenta. Eur J Immunol 29: 733-744, 1999 .

20. Heaton SJ, Eady JJ, Parker ML, et al: The use of BeWo cells as an in vitro model for placental iron transport. Am J Physiol Cell Physiol 295: 1445-1453, 2008.

21. Manley SW, Li H and Mortimer RH: The BeWo choriocarcinoma cell line as a model of iodide transport by placenta. Placenta 26 : 380-386, 2005

22. Bachmann RA, Kim JH, Wu AL, Park IH and Chen J: A nuclear transport signal in mammalian target of rapamycin is critical for its cytoplasmic signaling to S6 kinase 1. J Biol Chem 281: 7357-7363, 2006

23. Burleigh DW, Kendziorski CM, Choi YJ, Grindle KM, Grendell RL, Magness RR and Golos TG: Microarray analysis of BeWo and JEG3 trophoblast cell lines: identification of differentially expressed transcripts. Placenta. 28: 383-389, 2007. 